\title{
A New PCR-Based Assay for Testing Bronchoalveolar Lavage Fluid Samples from Patients with Suspected Pneumocystis jirovecii Pneumonia
}

\author{
Flora Marzia Liotti ${ }^{1,2,+}$, Brunella Posteraro ${ }^{1,3, \dagger}$, Giulia De Angelis ${ }^{1,2}$, Riccardo Torelli ${ }^{2}$, Elena De Carolis ${ }^{2}$, \\ Domenico Speziale ${ }^{2}$, Giulia Menchinelli ${ }^{1,2}$, Teresa Spanu ${ }^{1,2}$ and Maurizio Sanguinetti ${ }^{1,2, *}$ (1) \\ 1 Dipartimento di Scienze Biotecnologiche di Base, Cliniche Intensivologiche e Perioperatorie, \\ Università Cattolica del Sacro Cuore, Largo A. Gemelli 8, 00168 Roma, Italy; \\ floramarzialiotti@gmail.com (F.M.L.); brunella.posteraro@unicatt.it (B.P.); \\ giulia.deangelis78@gmail.com (G.D.A.); giulia.menchinelli@unicatt.it (G.M.); teresa.spanu@unicatt.it (T.S.) \\ 2 Dipartimento di Scienze di Laboratorio e Infettivologiche, Fondazione Policlinico Universitario A. Gemelli \\ IRCCS, Largo A. Gemelli 8, 00168 Roma, Italy; riccardo.torelli@policlinicogemelli.it (R.T.); \\ elena.decarolis@policlinicogemelli.it (E.D.C.); domenico.speziale@policlinicogemelli.it (D.S.) \\ 3 Dipartimento di Scienze Mediche e Chirurgiche, Fondazione Policlinico Universitario A. Gemelli IRCCS, \\ Largo A. Gemelli 8, 00168 Roma, Italy \\ * Correspondence: maurizio.sanguinetti@unicatt.it; Tel.: +39-06-3054-411; Fax: +39-06-3051-152 \\ check for \\ updates \\ $+\quad$ The first two authors contributed equally to this work.
}

Citation: Liotti, F.M.; Posteraro, B.; De Angelis, G.; Torelli, R.; De Carolis, E.; Speziale, D.; Menchinelli, G.; Spanu, T.; Sanguinetti, M. A New PCR-Based Assay for Testing Bronchoalveolar Lavage Fluid Samples from Patients with Suspected Pneumocystis jirovecii Pneumonia. J. Fungi 2021, 7, 681. https://doi.org/10.3390/jof7090681

Academic Editors: Enrique J. Calderón, Robert F. Miller and Yaxsier de Armas

Received: 21 July 2021

Accepted: 22 August 2021

Published: 24 August 2021

Publisher's Note: MDPI stays neutral with regard to jurisdictional claims in published maps and institutional affiliations.

Copyright: () 2021 by the authors. Licensee MDPI, Basel, Switzerland. This article is an open access article distributed under the terms and conditions of the Creative Commons Attribution (CC BY) license (https:// creativecommons.org/licenses/by/ $4.0 /)$.
Abstract: To support the clinical laboratory diagnosis of Pneumocystis jirovecii (PJ) pneumonia (PCP), an invasive fungal infection mainly occurring in HIV-negative patients, in-house or commercial PJ-specific real-time quantitative PCR (qPCR) assays are todays' reliable options. The performance of these assays depends on the type of $P J$ gene (multi-copy mitochondrial versus single-copy nuclear) targeted by the assay. We described the development of a PJ-PCR assay targeting the dihydrofolate reductase (DHFR)-encoding gene. After delineating its analytical performance, the PJ-PCR assay was used to test bronchoalveolar lavage (BAL) fluid samples from 200 patients (only seven were HIV positive) with suspected PCP. Of 211 BAL fluid samples, 18 (8.5\%) were positive and 193 (91.5\%) were negative by PJ-PCR. Of 18 PJ-PCR-positive samples, 11 (61.1\%) tested positive and seven (38.9\%) tested negative with the immunofluorescence assay (IFA). All (100\%) of the 193 PJ-PCR-negative samples were IFA negative. Based on IFA/PCR results, patients were, respectively, classified as having $(n=18)$ and not having $(n=182)$ proven $(P J-P C R+/ I F A+)$ or probable $(P J-P C R+/ I F A-)$ PCP. For 182 patients without PCP, alternative infectious or non-infectious etiologies were identified. Our PJ-PCR assay was at least equivalent to IFA, fostering studies aimed at defining a qPCR-based standard for PCP diagnosis in the future.

Keywords: BAL fluid; dihydrofolate reductase gene; Pneumocystis jirovecii pneumonia; qPCR assay

\section{Introduction}

Fungal infections, mainly Pneumocystis jirovecii (PJ; pneumocystosis), Aspergillus (aspergillosis), or Mucorales (mucormycosis), account for about $5 \%$ of all severe respiratory infections (including bacterial, mycobacterial, or viral infections) that occur in immunocompromised patients [1]. The non-specificity of either respiratory symptoms or imaging features necessitates differentiation among possible infectious etiologies as well as between infectious and non-infectious respiratory abnormalities [1]. Molecular-based methods for detecting pneumonia agents in multiple or single assays have been developed to overcome the limits of conventional (microscopy- or culture-based) identification methods, which are familiar in medical mycology [2] especially with PJ organisms [3].

While FDA-cleared molecular panels comprise a variety of detectable bacterial or viral agents from the lower respiratory tract (LRT) bronchoalveolar lavage (BAL) fluid-the 
preferred sample for PCP diagnosis [4] —or other samples [5], only one panel—to our knowledge-has recently comprised PJ [6]. Thus, it is unsurprising that in-house or commercial PJ-specific PCR assays, mostly real-time quantitative PCR (qPCR) assays, became reliable options to support today's clinical laboratory diagnosis of Pneumocystis pneumonia (PCP) [7]. Despite helping to distinguish colonization from infection, qPCR assays show variable performance [8], which depends on the type of PJ gene-multi-copy mitochondrial (i.e., mitochondrial small subunit (mtSSU) of ribosomal RNA) versus single-copy nuclear (e.g., beta-tubulin or dihydrofolate reductase (DHFR)) encoding gene- -targeted by the assay. However, the predictably best analytical sensitivity of the mtSSU gene may be detrimental when considering the risk for false-positive results. Notably, quantification of a single-copy gene may better reflect the $P J$ load-in terms of genomic copies $/ \mathrm{mL}$-in an LRT sample [8].

Here, we report on the detection of PJ DHFR DNA in BAL fluid samples, prospectively collected from patients hospitalized at our institution (a large tertiary care hospital in Rome, Italy), using a new in-house qPCR assay (hereafter referred to as PJ-PCR assay).

\section{Materials and Methods}

Between 1 March 2019 and 1 March 2020, patients hospitalized at the Fondazione Policlinico Universitario A. Gemelli IRCCS (Roma, Italy) with a sampled BAL fluid for microbiological examination were eligible for the study. We included samples from patients (age, $\geq 18$ years) with PCP- [9] or non-PCP [1]-compatible symptoms (e.g., cough, dyspnea, and hypoxemia) and radiological findings (e.g., bilateral ground-glass opacities) who had or did not have typical risk factors for PCP $[10,11]$. Before examination, BAL fluid samples were centrifuged and the resuspended pellets $(200 \mu \mathrm{L})$ were used for both $P J$-microscopy $(50 \mu \mathrm{L})$ and PCR assay $(100 \mu \mathrm{L})$, as specified below. All samples allowed one to extract enough human DNA to make $P J$ detectable in BAL fluid, based on the positive amplification of the human RNase gene (see below for details). This gene was equivalent to the human $\beta$-globin gene used elsewhere.

The immunofluorescence assay (IFA) of BAL fluid samples was performed using the MONOFLUO ${ }^{\mathrm{TM}}$ Pneumocystis jirovecii IFA Test kit (BioRad, Hercules, CA) to identify PJ asci and trophic forms at microscopy. Using the forward (5'-GGCTGATCAAAGAAGCATGGATA) or reverse (5'-CGGCATAGACATATTCGATACTTGTT) primer pair and the internal probe (5'-TGCGTGAAACAGATACATGGAGCTCTACCC) targeting the PJ DHFR-encoding gene (GenBank Accession number, DQ269976.1), we developed the PJ-PCR assay following Minimum Information for the publication of real-time Quantitative PCR Experiments (MIQE) guidelines [12].

To determine the PJ-PCR assay's performance, the full-length DHFR gene was amplified, the resulting PCR product was cloned into a pCR 2.1 cloning vector (Zero Blunt ${ }^{\circledR}$ PCR Cloning Kit, Invitrogen, Carlsbad, CA), and the recombinant plasmid DNA concentration was measured with the Qubit 4 fluorometer (Thermo Fisher Scientific, Waltham, MA). Using a 1:10 dilution series of DHFR-carrying plasmid (ranging from approximately $10^{-1}$ to $10^{9}$ copies $/ \mathrm{mL}$ ), a standard (calibration) curve was generated to calculate the amplification efficiency, which was established to be around $99 \%$. Specifically, using the equation PCR efficiency $=10^{-1 / \text { slope }}-1$, we plotted the logarithm of the initial DNA concentration on the $x$-axis and the quantification cycle $(\mathrm{Cq})$-also termed threshold cycle $(\mathrm{Ct})$-on the $y$-axis. Then, we determined the PJ-PCR assay's analytical sensitivity, expressed as the limit of detection (LoD), which was found to be equivalent to 10 copies $/ \mathrm{mL}$. The $\mathrm{Ct}$ variation (as assessed at $10 \mathrm{X} \mathrm{LoD}$ ) was 0.6 , whereas the PJ-PCR assay had $100 \%$ analytical specificity, as shown elsewhere [13]. Additionally, we verified that the PJ-PCR assay was stable by testing positive (DHFR-carrying plasmid) samples in qPCRs after the reagents were subjected to several freezing/thawing cycles.

As previously described [14,15], a diagnostic platform automatically extracted and dispensed microbial DNA into qPCR reagents' prefilled microwell strips before manually loading them onto the BioRad CFX Thermal Cycler (BioRad, Hercules, CA, USA) qPCR 
instrument. Primers' and probe's concentrations were $400 \mathrm{nM}$ or $150 \mathrm{nM}$, respectively, whereas thermal cycling conditions consisted of an initial denaturation step at $95^{\circ} \mathrm{C}$ for $8 \mathrm{~min}$, followed by 50 cycles of $95^{\circ} \mathrm{C}$ for $15 \mathrm{~s}$ and $60^{\circ} \mathrm{C}$ for $1 \mathrm{~min}$. For each sample, the number of amplification cycles required to produce a positive signal for the DHFR gene was expressed as a Ct value; thus, samples with $\mathrm{Ct}$ values $\leq 40$ or $>40$ were scored as $P J$-PCR positive or negative, respectively. In each run, the aforementioned human RNase gene was included as an external intrinsic control to monitor PCR inhibition, and no-template (sterile water) controls were included to monitor PCR contamination.

In parallel, patients' serum samples (collected the same day or within \pm one day of BAL fluid sampling) were tested with the Fungitell ${ }^{\circledR}$ assay (Associates of Cape Cod, East Falmouth, MA, USA) for 1,3- $\beta$-D-glucan (BDG) measurement using a positivity cutoff of $80 \mathrm{pg} / \mathrm{mL}[11]$.

According to positive IFA or PJ-PCR results, the revised/updated EORTC/MSGERC 2008 consensus definitions of invasive fungal disease [11] allowed one to classify pneumonia episodes as proven (both IFA and PJ-PCR positive) or probable (only PJ-PCR positive) PCP episodes, respectively. In view of conflicting opinions about the role of BDG in the PCP diagnosis $[4,16,17]$, we only used BDG results to corroborate the results obtained with the PJ-PCR assay. Furthermore, microbiological standard-of-care testing results allowed one to classify non-PCP episodes as bacterial, viral, or (outside PCP) fungal pneumonia episodes, respectively.

\section{Results}

As shown in Figure 1, 200 patients with their BAL fluid samples $(n=211)$ were investigated for PCP. Based on both PJ-PCR and IFA results, patients were classified as having $(n=18)$ or not having $(n=182)$ proven $(\mathrm{PCR}+/ \mathrm{IFA}+)$ or probable $(\mathrm{PCR}+/ \mathrm{IFA}-)$ PCP [11]. Samples that tested positive with both PCR and IFA had Ct values lower than in samples that tested positive with PCR and negative with IFA.

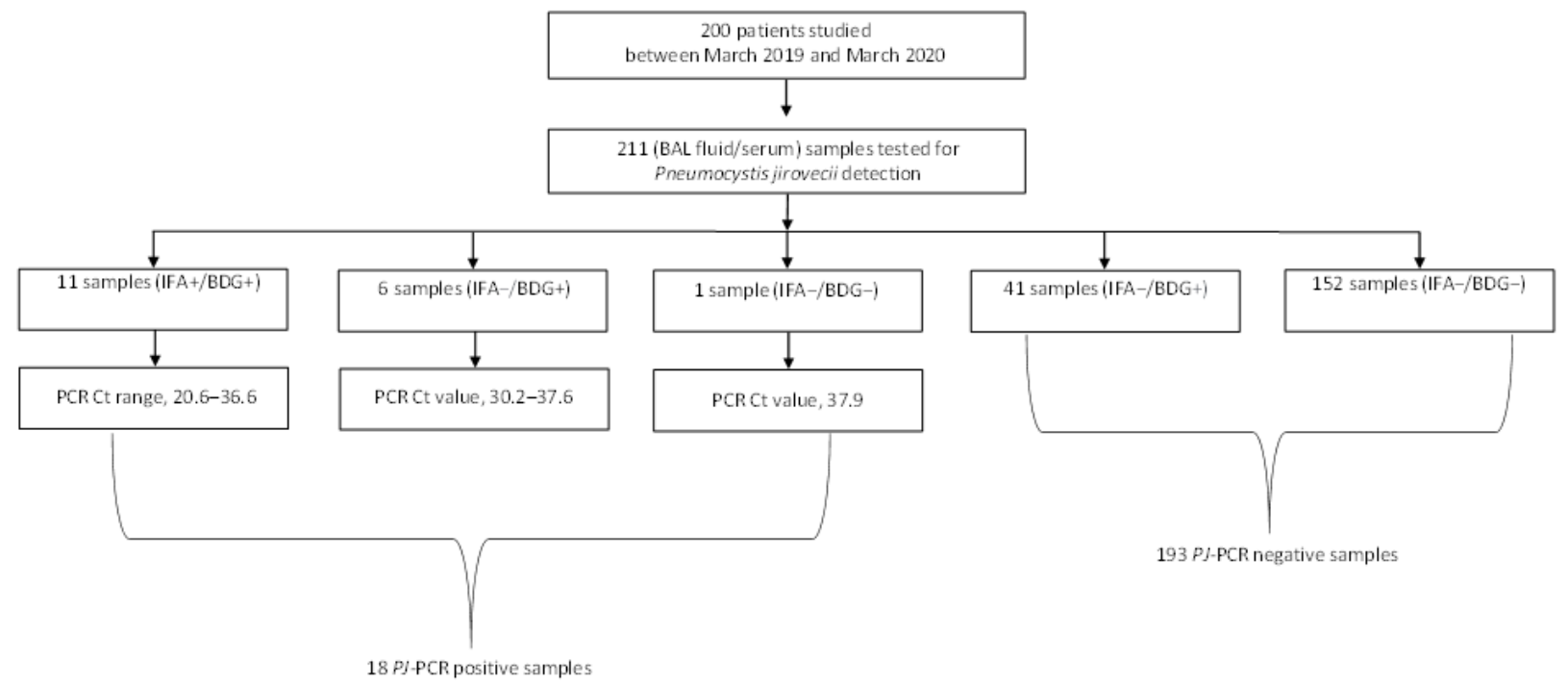

Figure 1. Overview of samples obtained from 200 patients with suspicion of PCP that underwent clinical laboratory testing. Bronchoalveolar lavage (BAL) fluid samples were tested by both PJ-PCR and IFA, whereas serum samples, which were obtained almost concomitantly to BAL fluid sampling, were tested for BDG. For PJ-PCR positive samples, PCR Ct values are shown according to the positivity ( 11 samples) or negativity ( 7 samples) by IFA.

Table 1 summarizes PJ-PCR/IFA results for 211 pneumonia episodes, 191 of which were single and 20 were multiple. One patient had four episodes and eight patients had each two episodes. Of 211 BAL fluid samples studied in total, $18(8.5 \%)$ tested positive (Ct values, 20.6-37.9) and 193 (91.5\%) tested negative (Ct values, $>40)$ with the PJ-PCR assay. 
Table 1. Microbiological results for 211 BAL fluid samples from patients with suspected PCP.

\begin{tabular}{|c|c|c|c|}
\hline Patient Characteristics & $\begin{array}{c}\mathrm{PCR}+(\mathrm{Ct}, 30.6 \pm 4.4)^{\mathrm{a}} \& \mathrm{IFA}+ \\
(n=11)\end{array}$ & $\begin{array}{c}\text { PCR+ }(C t, 35.7 \pm 2.6)^{a} \& \text { IFA- } \\
(n=7)\end{array}$ & $\begin{array}{c}\text { PCR }-\& \text { IFA- } \\
\quad(n=193)\end{array}$ \\
\hline \multicolumn{4}{|l|}{ Underlying diseases/conditions } \\
\hline Hematological malignancy & 3 & 1 & 63 \\
\hline Inflammatory/rheumatic disease & 3 & 4 & 51 \\
\hline $\mathrm{SOT} / \mathrm{HSCT}^{\mathrm{b}}$ & 0 & 1 & 31 \\
\hline Solid malignancy & 2 & 0 & 17 \\
\hline HIV infection & 3 & 1 & 3 \\
\hline Other disease / condition ${ }^{c}$ & 0 & 0 & 28 \\
\hline $\begin{array}{l}\text { Chest X-ray/CT findings } \\
\text { Ground-glass opacity (GGO) }\end{array}$ & & & \\
\hline $\begin{array}{l}\text { Ground-glass opacity (GGU) } \\
\text { Nodules }\end{array}$ & $\begin{array}{l}5 \\
0\end{array}$ & $\begin{array}{l}2 \\
3\end{array}$ & $\begin{array}{l}10 \\
7\end{array}$ \\
\hline Consolidation & $\begin{array}{l}0 \\
3\end{array}$ & $\begin{array}{l}3 \\
2\end{array}$ & 112 \\
\hline Other findings ${ }^{d}$ & 3 & 0 & 64 \\
\hline \multicolumn{4}{|l|}{$\begin{array}{l}\text { Infection/colonization due to } \\
\text { organisms other than }\end{array}$} \\
\hline Pneumocystis jirovecii $\mathrm{e}$ & & & \\
\hline Bacteria & $4^{\mathrm{f}}$ & $3 \mathrm{~g}$ & 67 \\
\hline Viruses & 0 & 0 & 43 \\
\hline Aspergillus & 0 & $1^{\mathrm{h}}$ & 7 \\
\hline Candida & 0 & $1^{\mathrm{i}}$ & 27 \\
\hline None & 7 & 3 & $63^{j}$ \\
\hline
\end{tabular}

${ }^{\text {a }}$ Results by Pneumocystis jirovecii (PJ)-PCR on bronchoalveolar lavage (BAL) fluid samples are expressed as the mean threshold cycle $(\mathrm{Ct})$ value \pm standard deviation. Conversely, immunofluorescence assay (IFA) results on the same samples are expressed as positive or negative only. ${ }^{\mathrm{b}}$ SOT, solid organ transplantation; HSCT, hematopoietic stem cell transplantation. ${ }^{\mathrm{c}}$ Includes patients for whom the diagnostic workup comprised $P J$ testing in spite of their apparent lack of underlying conditions/factors known to affect or enhance the exposure to PCP [10]. Twenty-three of these patients were in the intensive care unit (ICU) from the hospital. ${ }^{\mathrm{d}}$ Includes interstitial infiltrates, atelectasis, pleural effusion, or pneumothorax, as assessed by chest X-ray or computed tomography (CT). ${ }^{\text {e }}$ Assessed by semiquantitative culture-, antigen-, or molecular-based standard-of-care testing methods. Some patients were concomitantly infected/colonized by more than one of the listed organisms. With regard to Candida organisms, 26 of 28 patients with culture-positive samples were deemed to be colonized by Candida species, whereas two remaining patients had a concomitant bloodstream infection caused by Candida albicans and, thus, were deemed to be infected by Candida species. ${ }^{\mathrm{f}}$ Includes Haemophilus influenzae (1 sample), Legionella pneumophila (1 sample), methicillin-resistant Staphylococcus aureus (1 sample), and methicillin-susceptible S. aureus (1 sample). ${ }^{g}$ Include Escherichia coli (1 sample), Mycobacterium tuberculosis (1 sample), and Pseudomonas aeruginosa plus Stenotrophomonas maltophilia (1 sample). ${ }^{\mathrm{h}}$ The sample was positive for Aspergillus fumigatus. I The sample was positive for Candida albicans. ${ }^{j}$ In these episodes, non-infectious etiologies of pneumonia included lung infiltration from the underlying disease, drug-related lung toxicity, etc.

Among 18 PJ-PCR-positive samples, 11 (61.1\%) samples (Ct values, 20.6-36.6) were IFA positive and seven (38.9\%) samples (Ct values, 30.2-37.9) were IFA negative. A total of $193(100 \%)$ of 193 PJ-PCR negative samples were IFA negative. Seventeen $(94.4 \%)$ of eighteen patients with PJ-PCR-positive BAL fluid samples had BDG-positive (315 to $>500 \mathrm{pg} / \mathrm{mL}$ ) serum samples, whereas one patient with a PJ-PCR-positive (Ct value, 37.9 ) but IFA-negative BAL fluid sample had a BDG-negative $(<80 \mathrm{pg} / \mathrm{mL})$ serum sample. The last patient was a hematological patient for whom a BAL fluid culture yielded positive results for both Pseudomonas aeruginosa and Stenotrophomonas maltophilia.

In 41 (21.2\%) of 193 PJ-PCR/IFA-negative episodes (Figure 1), all patients (36 of whom were immunocompromised) had BDG-positive ( 82 to $>500 \mathrm{pg} / \mathrm{mL}$ ) serum samples, and their BAL fluid samples were culture positive for Candida (16 samples), Aspergillus (two samples), and/or bacterial species (15 samples). Seven of eighteen patients with PCP were co-infected with bacteria (e.g., Legionella pneumophila, Mycobacterium tuberculosis, etc.) and two other patients were colonized with Aspergillus or Candida (Table 1). Among 182 patients without PCP, infectious (bacterial, viral, Candida, or Aspergillus) or non-infectious etiologies were identified (Table 1).

\section{Discussion}

The PCP diagnosis remains particularly challenging in HIV-negative immunocompromised or other seriously ill patients, who accounted for $96.5 \%(193 / 200)$ of patients in this study. Only 7 (3.5\%) of 200 patients were HIV positive. Excluding 28 episodes from patients with lower suspicion of disease (Table 1), our PJ-PCR assay allowed one to diagnose PCP in 18 (9.8\%) of 183 episodes from patients who, otherwise, were at high risk of developing disease. In addition to meeting clinical and radiological criteria, these patients had at least one of the EORTC/MSGERC designed host factors [11]. Besides being 
highly specific [13], our assay appeared to be highly sensitive for detecting PJ DNA in BAL fluid samples. All (100\%) of the 193 episodes with a negative PCR result were from patients who had a microbiological (i.e., non-PJ respiratory infection) or non-microbiological (e.g., non-infectious respiratory abnormality) reason on which the suspicion of PCP was initially based.

The detection of PJ microscopically in the BAL fluid (or pulmonary tissue) using conventional (Gomori methenamine silver) or immunofluorescence (specific fluorescent antibody) staining is the current diagnostic criterion for proven PCP [11]. We found that none of samples with a positive IFA result was negative by the PJ-PCR assay, whereas seven samples with a positive PCR result were negative by the IFA. These findings would be consistent with the lower $C t$ values observed in these samples, which might reflect a smaller burden of $P J$ organisms, thus resulting in false-negative results at the microscopic examination. Six of seven patients were patients not living with HIV (Table 1), which is a condition notoriously associated with a larger burden of $P J$ organisms in respiratory samples [3]. Therefore, we did not interpret the seven IFA negative results as falsely PCR positive results but as the results of patients who had benefited from the molecular rather than the microscopic diagnosis of PCP [4].

It is important to recall that, regardless of the type of PCR format employed for testing, a positive or negative PCR result in the BAL fluid should be interpreted according to strict diagnostic criteria [4]. These include clinical, radiological, and laboratory abnormalities that, unfortunately, may suggest different pneumonia etiologies as underlined above. Therefore, a PCR assay-especially a qPCR assay-is the right adjunct to a multifaceted workup to assist the diagnosis of PCP or other invasive fungal infections [1]. Consistently, 2020 EORTC/MSGERC invasive fungal disease definitions consider a PCR-positive respiratory sample (or a BDG-positive serum sample) as the only mycological evidence for probable PCP [11]. In agreement with the PCR assay, 17 patients with PCR-positive BAL fluid samples had serum samples that tested positive for BDG. In disagreement with the PCR assay, serum samples were BDG-positive in 41 patients with PCR-negative BAL fluid samples. Thus, our findings support the EORTC/MSGERC recommendations that require $\geq 2$ consecutive serum samples to be BDG positive to diagnose probable PCP if other etiologies have been ruled out [11]. Conversely, a negative BDG result-that may be potentially false negative because of a low PJ load and low BDG release [16] — strongly suggested the absence of PCP in our patients, which was supported by either the BAL fluid sample PCR negativity or the determination of alternative etiologies (Table 1).

Implementing a PJ qPCR assay in the clinical microbiology laboratory may have particular value in the context of the ongoing pandemic due to the severe acute respiratory syndrome coronavirus 2 (SARS-CoV-2) that causes coronavirus disease 2019 (COVID-19), because PJ-similar to other fungi (Aspergillus, Candida, or Mucorales) - has emerged as a notable coinfection agent in COVID-19 patients [18]. At the time of writing, the PJ-PCR assay was integrated into our clinical laboratory's diagnostic respiratory workflow, which includes multiplex PCR-based panels newly commercialized to detect bacterial and viral agents of syndromic infections [5]. As shown in this study (and before its integration in the laboratory), the assay underwent validation to clearly delineate its technical performance, including the LoD and the amplification yield or control [12].

With regard to PJ qPCR assays, the choice of target genes for amplification remains an open issue [7]. Recently, Huh et al. [19] showed the similarity of two commercial assaysone of which was capable of amplifying the mitochondrial large subunit (mtLSU) of a ribosomal RNA gene-in detecting low PJ loads in BAL fluids. Very recently, Dellière et al. [20] found that an automated commercial reverse-transcriptase qPCR ( $2.2 \mathrm{~h}$ workflow) was more sensitive than their in-house qPCR assays ( $\sim 5 \mathrm{~h}$ workflow), which amplified both $\mathrm{mtSSU}$ and mtLSU for detecting PJ in respiratory samples. Therefore, the use of DHFR in our study might be questionable because of the (expected) lower sensitivity of a single-copy nuclear gene compared to multi-copy mitochondrial genes [8]. However, our study is reminiscent of a strategy reported by Huggett et al. [21] in 2008. Unlike us, the authors 
used the $P J$ heat shock protein 70 (HSP70) gene to detect PJ DNA in BAL fluid samples of HIV-positive patients (132 in total; only 7 were HIV positive in our study). The analytical sensitivity of their HSP70 real-time assay was $\sim$ five copies/reaction, but a cutoff value of $\sim 10$ copies/reaction was used to show (based on receiver-operator curve analysis) a clinical sensitivity of $98 \%$ and specificity of $96 \%$ for the diagnosis of PCP [21]. Furthermore, the authors [21] optimized the DNA-extraction PCR step that, if suboptimal, makes a negative PCR result difficult to interpret. We carefully assessed the sample-processing step (i.e., extraction) so that the PJ-PCR assay developed by us could represent the natural expansion of an existing molecular diagnostic platform in our laboratory [14], which is now capable of amplifying unique, species-specific genes using DNA automatically extracted from BAL fluid (or other clinical) samples ( $\sim 4 \mathrm{~h}$ workflow). Comparing our in-house assay with commercially available or, specifically, mtSSU- or mtLSU-based assays for $P J$ detection $[7,8]$ was beyond the scope of the present study. In essence, we sought to develop a qPCR assay that was at least equivalent to the IFA and that, ultimately, allowed us to replace microscopic with molecular testing for PCP diagnosis.

In conclusion, our PJ-PCR assay proved to be a sensitive and specific diagnostic tool for $P J$ infection in BAL fluid samples, thus emphasizing the increasing role of $P J$ qPCR assays as a clinical laboratory strategy to diagnose PCP in HIV and non-HIV patients [3,7]. We hope that our assay enters large-scale evaluation studies aimed at defining the performance of a standard qPCR-based method for the diagnosis of PCP.

Author Contributions: Conceptualization, F.M.L., B.P. and M.S.; investigation, F.M.L., G.D.A., R.T., E.D.C. and D.S.; analysis, F.M.L., B.P. and G.D.A.; data curation, D.S., G.M., T.S. and M.S.; resources, M.S.; writing-original draft, B.P. writing—review and editing, F.M.L., G.M., B.P. and M.S.; supervision, T.S. and M.S. All authors have read and agreed to the published version of the manuscript.

Funding: This research was supported by the Università Cattolica del Sacro Cuore (UCSC), Roma, Italy (research grant Linea D1). This implies that UCSC had no role in the design of the study; in the collection, analyses, or interpretation of data; in the writing of the manuscript, or in the decision to publish the results.

Institutional Review Board Statement: The institutional Ethics Committee approved the study (protocol code 0008175/19; date of approval, 20 February 2019).

Informed Consent Statement: Informed consent was obtained from all subjects involved in the study.

Acknowledgments: We are indebted to Franziska Michaela Lohmeyer for providing English language assistance. The study was presented in part at the 29th European Congress of Clinical Microbiology and Infectious Diseases (ECCMID) in Amsterdam, Netherlands, 13-16 April 2019.

Conflicts of Interest: The authors declare no conflict of interest.

\section{References}

1. Azoulay, E.; Russell, L.; Van de Louw, A.; Metaxa, V.; Bauer, P.; Povoa, P.; Montero, J.G.; Loeches, I.M.; Mehta, S.; Puxty, K.; et al. Diagnosis of severe respiratory infections in immunocompromised patients. Intensive Care Med. 2020, 46, 298-314. [CrossRef] [PubMed]

2. Wickes, B.L.; Wiederhold, N.P. Molecular diagnostics in medical mycology. Nat. Commun. 2018, 9, 5135. [CrossRef]

3. Bateman, M.; Oladele, R.; Kolls, J.K. Diagnosing Pneumocystis jirovecii pneumonia: A review of current methods and novel approaches. Med. Mycol. 2020, 58, 1015-1028. [CrossRef]

4. Alanio, A.; Hauser, P.M.; Lagrou, K.; Melchers, W.J.; Helweg-Larsen, J.; Matos, O.; Cesaro, S.; Maschmeyer, G.; Einsele, H.; Donnelly, J.P.; et al. ECIL guidelines for the diagnosis of Pneumocystis jirovecii pneumonia in patients with haematological malignancies and stem cell transplant recipients. J. Antimicrob. Chemother. 2016, 71, 2386-2396. [CrossRef] [PubMed]

5. Ramanan, P.; Bryson, A.L.; Binnicker, M.J.; Pritt, B.S.; Patel, R. Syndromic panel-based testing in clinical microbiology. Clin. Microbiol. Rev. 2017, 31, e00024-17. [CrossRef]

6. Klein, M.; Bacher, J.; Barth, S.; Atrzadeh, F.; Siebenhaller, K.; Ferreira, I.; Beisken, S.; Posch, A.E.; Carroll, K.C.; Wunderink, R.G.; et al. Multicenter evaluation of the Unyvero platform for testing bronchoalveolar lavage fluid. J. Clin. Microbiol. 2021, 59, e02497-20. [CrossRef] [PubMed]

7. Kidd, S.E.; Chen, S.C.; Meyer, W.; Halliday, C.L. A new age in molecular diagnostics for invasive fungal disease: Are we ready? Front. Microbiol. 2020, 10, 2903. [CrossRef] [PubMed] 
8. Gits-Muselli, M.; White, P.L.; Mengoli, C.; Chen, S.; Crowley, B.; Dingemans, G.; Fréalle, E.; Gorton, R.L.; Guiver, M.; Hagen, F.; et al. The Fungal PCR Initiative's evaluation of in-house and commercial Pneumocystis jirovecii qPCR assays: Toward a standard for a diagnostics assay. Med. Mycol. 2020, 58, 779-788. [CrossRef]

9. Thomas, C.F., Jr.; Limper, A.H. Pneumocystis pneumonia. N. Engl. J. Med. 2004, 350, 2487-2498. [CrossRef]

10. Sokulska, M.; Kicia, M.; Wesołowska, M.; Hendrich, A.B. Pneumocystis jirovecii_From a commensal to pathogen: Clinical and diagnostic review. Parasitol. Res. 2015, 114, 3577-3585. [CrossRef] [PubMed]

11. Donnelly, J.P.; Chen, S.C.; Kauffman, C.A.; Steinbach, W.J.; Baddley, J.W.; Verweij, P.E.; Clancy, C.J.; Wingard, J.R.; Lockhart, S.R.; Groll, A.H.; et al. Revision and update of the consensus definitions of invasive fungal disease from the European Organization for Research and Treatment of Cancer and the Mycoses Study Group Education and Research Consortium. Clin. Infect. Dis. 2020, 71, 1367-1376. [CrossRef]

12. Bustin, S.A.; Benes, V.; Garson, J.A.; Hellemans, J.; Huggett, J.; Kubista, M.; Mueller, R.; Nolan, T.; Pfaffl, M.W.; Shipley, G.L.; et al. The MIQE guidelines: Minimum information for publication of quantitative real-time PCR experiments. Clin. Chem. 2009, 55, 611-622. [CrossRef] [PubMed]

13. Bandt, D.; Monecke, S. Development and evaluation of a real-time PCR assay for detection of Pneumocystis jirovecii. Transpl. Infect. Dis. 2007, 9, 196-202. [CrossRef] [PubMed]

14. Liotti, F.M.; Posteraro, B.; Mannu, F.; Carta, F.; Pantaleo, A.; De Angelis, G.; Menchinelli, G.; Spanu, T.; Fiori, P.L.; Turrini, F.; et al. Development of a multiplex PCR platform for the rapid detection of bacteria, antibiotic resistance, and Candida in human blood samples. Front. Cell. Infect. Microbiol. 2019, 9, 389. [CrossRef]

15. Liotti, F.M.; De Angelis, G.; Speziale, D.; Morandotti, G.A.; Genovese, O.; Sanguinetti, M.; Posteraro, B. Bordetella pertussis DNA detected in a tracheostomized child blood before seroconversion. Eur. J. Clin. Microbiol. Infect. Dis. 2021, 40, 205-208. [CrossRef]

16. Mercier, T.; Aissaoui, N.; Gits-Muselli, M.; Hamane, S.; Prattes, J.; Kessler, H.H.; Mareković, I.; Pleško, S.; Steinmann, J.; Scharmann, U.; et al. Variable correlation between bronchoalveolar lavage fluid fungal load and serum-(1,3)- $\beta$-D-glucan in patients with pneumocystosis-A multicenter ECMM Excellence Center study. J Fungi 2020, 6, 327. [CrossRef]

17. Del Corpo, O.; Butler-Laporte, G.; Sheppard, D.C.; Cheng, M.P.; McDonald, E.G.; Lee, T.C. Diagnostic accuracy of serum (1-3)- $\beta$-D-glucan for Pneumocystis jirovecii pneumonia: A systematic review and meta-analysis. Clin. Microbiol. Infect. 2020, 26, 1137-1143. [CrossRef]

18. Chong, W.H.; Saha, B.K.; Chopra, A. Narrative review of the relationship between COVID-19 and PJP: Does it represent coinfection or colonization? Infection 2021, 1-12. [CrossRef]

19. Huh, H.J.; Lim, K.R.; Ki, C.S.; Huh, K.; Shim, H.J.; Song, D.J.; Kim, Y.J.; Chung, D.R.; Lee, N.Y. Comparative evaluation between the RealStar Pneumocystis jirovecii PCR Kit and the AmpliSens Pneumocystis jirovecii (carinii)-FRT PCR Kit for detecting P. jirovecii in non-HIV immunocompromised patients. Ann. Lab. Med. 2019, 39, 176-182. [CrossRef] [PubMed]

20. Dellière, S.; Hamane, S.; Aissaoui, N.; Gits-Muselli, M.; Bretagne, S.; Alanio, A. Increased sensitivity of a new commercial reverse transcriptase-quantitative PCR for the detection of Pneumocystis jirovecii in respiratory specimens. Med. Mycol. 2021, 59, 845-848. [CrossRef]

21. Huggett, J.F.; Taylor, M.S.; Kocjan, G.; Evans, H.E.; Morris-Jones, S.; Gant, V.; Novak, T.; Costello, A.M.; Zumla, A.; Miller, R.F. Development and evaluation of a real-time PCR assay for detection of Pneumocystis jirovecii DNA in bronchoalveolar lavage fluid of HIV-infected patients. Thorax 2008, 63, 154-159. [CrossRef] [PubMed] 